\title{
Color aftereffects contingent on perceived spatial frequency
}

\author{
KEVIN JORDAN \\ Quincy College, Quincy, Illinois 62301 \\ and \\ JOHN UHLARIK \\ Kansas State University, Manhattan, Kansas 66506
}

\begin{abstract}
Spatial frequency-contingent color aftereffects were induced by adaptation to a single color grating of a fixed spatial frequency (Experiment 1) or by alternate exposure to two different color gratings of two different spatial frequencies (Experiment 2). The test stimuli for both experiments consisted of gratings placed in two-dimensional pictorial arrays (Necker cube and corridor array) such that although the retinal spatial frequencies of the test gratings were identical, the perceived spatial frequencies were different due to implicit depth relations and size constancy. The results indicated that under these conditions the color aftereffects were contingent on perceived rather than retinal spatial frequency.
\end{abstract}

The present experiments examined the possibility that color aftereffects can be shown to be contingent on perceived spatial frequency without accompanying changes in retinal spatial frequency. These factors were separated by using test stimuli in which retinal spatial frequency was held constant while perceived spatial frequency varied due to pictorial depth cues. Two pictorial arrays that illustrate this effect are the Necker cube and the receding corridor array shown in Figures 1 and 2 . These arrays were chosen as a context in which to present test gratings of identical retinal spatial frequency in an attempt to demonstrate color aftereffects contingent on perceived spatial frequency. For example, although both gratings in Figures 1 and 2 are of identical retinal spatial frequency, the depth cues make one of the gratings in each array appear to be more distant, which should give rise to differences in perceived size and, hence, in perceived spatial frequency.

\section{EXPERIMENT 1}

Individual observers were adapted to a single grating of a single color (magenta or green) and spatial frequency ( 3 or 6 cycles/deg). Observers were then tested for a color aftereffect contingent on perceived spatial frequency using both the Necker cube and the corridor array. Each test array contained two achromatic gratings of identical retinal spatial frequency (i.e., 4.2 cycles/deg). Thus, depending on the spatial frequency of the adapting grating, the test gratings were either .5 octave higher or .5 octave lower than the adapting spatial frequency.

Requests for reprints should be sent to John Uhlarik, Department of Psychology, Kansas State University, Manhattan, Kansas 66506.

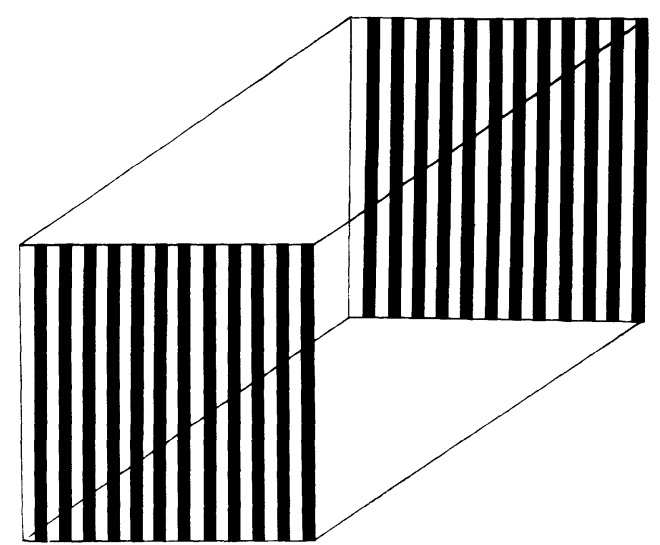

Figure 1. Example of a Necker cube that manifests Emmert's law. The two gratings are of identical retinal spatial frequency.

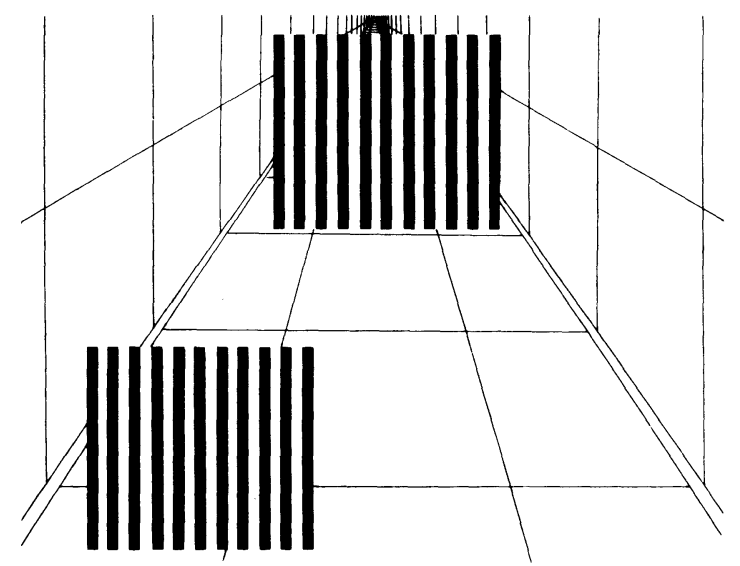

Figure 2. Example of a receding corridor array that manifests Emmert's law. The two gratings are of identical retinal spatial frequency. 
Even though there was a slight mismatch between the adapting and test frequencies ( 3 or 6 and 4.2 cycles $/ \mathrm{deg}$ ), frequency-contingent aftereffects are typically reported over 4 octaves; the closer the match, the stronger is the aftereffect (Stromeyer, 1972). If the aftereffect is contingent only on retinal spatial frequency, the strength of the aftereffect should be identical (i.e., equally saturated) for both of the 4.2-cycle/deg test gratings contained in each of the arrays. However, if the arrays are perceived in depth due to pictorial cues, the grating in that region of the array perceived as more distant would be perceived as larger than the relatively closer grating due to Emmert's law. Therefore, if perceived spatial frequency of the test gratings influences contingent aftereffects, the test grating that appears to be the closer match in spatial frequency to the adapting grating should evoke a stronger color aftereffect than the apparently more disparate test grating.

\section{Method}

Observers. Twenty-four undergraduate students participated in the experiment in order to fulfill a psychology course requirement. None of the observers were familiar with color aftereffects, and all had normal red-green color vision, as determined by pseudoisochromatic plates.

Stimuli and Design. All stimuli were $35-\mathrm{mm}$ slides projected on the rear of a translucent screen. Presentation of adaptation stimuli was controlled by a two-channel projection tachistoscope (Marietta No. 15-5-C). The adaptation stimuli consisted of square-wave gratings photographed on Kodak high-contrast (No. 5069) copy film. The projected image of the adaptation and test slides subtended a visual angle of 4 deg horizontally and $2.6 \mathrm{deg}$ vertically at a viewing distance of $2.5 \mathrm{~m}$. There were four adaptation conditions determined by the factorial combination of two spatial frequencies ( 3 or 6 cycles/deg) projected through either a magenta (Wratten No. 34a) or green (Wratten No. 53) gelatin filter. Adaptation condition was a between-subjects factor, and observers were randomly assigned to one of the four conditions, with the restriction that there be six observers in each condition. The adaptation gratings were always oriented vertically. The luminances of the black bars in combination with the magenta slits were 2 and $31 \mathrm{~mL}$, respectively; the luminances of the black bars and green slits were 5 and $143 \mathrm{~mL}$, respectively.

A composite grating used as a preliminary test for spatial frequency-contingent color aftereffects contained achromatic vertical 3-cycle/deg square waves on the left and 6-cycle/deg square waves on the right. In addition, 4.2-cycle/deg vertical square-wave gratings were presented in the context of the Necker cube and receding corridor array to test for color aftereffects contingent on perceived spatial frequency (see Figures 1 and 2). The luminances of the black bars and light slits of all test gratings were 30 and $447 \mathrm{~mL}$, respectively.

Procedure. Each observer was adapted for $30 \mathrm{~min}$ to a grating of a single color and spatial frequency. The adapting grating was presented for $25 \mathrm{sec}$ and alternated with 5 -sec dark periods.

The observers rested for $10 \mathrm{~min}$ after adaptation to allow any afterimages to dissipate. Subsequently, observers were systematically questioned to determine (1) if spatial frequencycontingent color aftereffects obtained, (2) whether these color aftereffects were contingent on perceived or retinal spatial frequency, and (3) if the perceived sizes of the test gratings on the Necker cube and corridor array were consistent with Emmert's law. The following questions, with the exception of the first question (A1), were specified as two-alternative forced choices.

(A) Preliminary test for a spatial frequency-contingent color aftereffect (achromatic vertical 3-cycle/deg square waves on the left and 6-cycle/deg square waves on the right)-(1) Do you see any trace of color on this slide? If so, what color? (2) If y ou had to say that either the left or the right side of this slide contained more of this color, which would it be, left or right?

(B) Tests for color aftereffects contingent on perceived spatial frequency-Necker cube: (1) Which face of this cube appears to be the front, lower left or upper right? (2) While the cube is in this organization, if you had to say that one of the gratings contained more color than the other, which would it be, lower left or upper right? (3) Now try to reverse the organization of the cube. Which face of the cube appears to be the front, lower left or upper right? (4) While the cube is in this organization, if you had to say that one of the gratings contained more color than the other, which would it be, lower left or upper right? Corridor array: (5) If you had to say that one of the gratings on this slide contained more color than the other, which would it be, lower left or upper right?

(C) Tests for perceived size differences between the gratingsCorridor array: (1) If you had to say that one of the gratings on this slide was larger than the other, which would it be, lower left or upper right? Necker cube: (2) Which face of this cube appears to be the front, lower left or upper right? (3) While the cube is in this organization, if you had to say that one grating was larger than the other, which would it be, lower left or upper right?

\section{Results and Discussion}

In order to be included in the test phase of the experiment, each observer had to report a color that was approximately complementary to the color of the adapting grating in response to Question $\mathrm{A} 1$. In addition, the response to Question A2 had to indicate that the aftereffect was strongest in that region of the preliminary test pattern with the same spatial frequency as the adapting grating. Eleven observers were replaced in the appropriate adaptation conditions for failing to meet these prerequisites.

There were no systematic differences among adaptation conditions in the pattern of responding to the postadaptation questions in Parts $\mathrm{B}$ and $\mathrm{C}$, so these results were pooled across conditions. Because of its reversible organization, either face of the Necker cube could be reported as the front in response to Question B1. Regardless of the observer's response to Question B1, whichever face was organized as front should be perceived as smaller (i.e., higher spatial frequency) than the other (back) face due to implied depth relations. If perceived factors influence contingent color aftereffects, the low-spatial-frequency adapting grating (i.e., 3 cycles/ deg) would be expected to produce the most saturated aftereffect on the test grating that appeared to be of the lower spatial frequency. The high-spatial-frequency adaptation condition (i.e., 6 cycles/deg) would be expected to produce the most saturated aftereffect on the grating that appeared to be of the higher spatial frequency. On the other hand, if the aftereffect was based solely on retinal spatial frequency, responding to the two-alternative forced-choice questions would have been random, with chance performance being 12 of 24 observers choosing any given alternative.

Eighteen of the 24 observers gave a response to Question B2 that was consistent with an aftereffect contingent on perceived spatial frequency of the test 
gratings on the Necker cube $(\mathrm{p}<.05) .{ }^{1}$ However, when the observers were asked to reverse the organization of the cube (Question B3) and then report which grating appeared more saturated (Question B4), only 10 of the 24 observers gave a response consistent with a perceived spatial frequency-contingent aftereffect. A reversal of differential aftereffect color with a reversal of cube organization would have provided additional support for the contingency of color aftereffects on perceived spatial frequency. However, most observers reported difficulty reversing the perceptual organization after noting differential aftereffect color in the previous comparison (Question B2).

The final color judgment (Question B5) tested for differential strength of the aftereffect for the two gratings contained in the corridor array. Since this array involves fixed depth relations, the upper grating should always be perceived as larger (i.e., lower spatial frequency) than the lower grating. The response to Question B5, consistent with an aftereffect contingent on perceived spatial frequency, was the test grating that was the closest perceptual match to the spatial frequency of the adapting grating. Nineteen of the 24 observers $(p<.05)$ responded in this manner.

Twenty-one of the 24 observers $(p<.01)$ gave a response to Question $\mathrm{C} 1$ regarding perceived size differences between the two test gratings on the corridor array that was consistent with Emmert's law. In addition, 18 of the 24 observers $(p<.05)$ gave a response to Question C3 regarding perceived size differences between the two test gratings on the Necker cube consistent with Emmert's law. These results indicate that there were in fact perceived size differences between the test gratings, which supports the underlying assumption on which the questions in Part B were based.

Taken together, the color and size judgments provide support for the hypothesis that the nature of color aftereffects can be influenced by the perceived spatial frequency of test gratings.

\section{EXPERIMENT 2}

In this experiment, color aftereffects were examined by adapting observers to the alternate exposure of two different chromatic gratings (magenta and green) separated by 1 octave of spatial frequency ( 3 and 6 cycles/ deg). Observers were then tested for aftereffects contingent on perceived spatial frequency using Figures 1 and 2 .

If the test gratings contained on the Necker cube and the corridor array are characterized by differences in perceived depth, it is not possible to describe a priori the location of these gratings in space relative to the picture plane. In order to control for the possibility that individual observers used different relative reference points in the pictorial arrays, subjects were allowed to adjust the distance between themselves and the viewing screen during the test phase of Experiment 2. By doing so, observers should be able to find an optimal point (distance) at which one grating in the test array is perceived as more similar in spatial frequency to one of the adapting gratings and the other test grating is perceived as more similar to the other adapting grating.

An additional dependent measure obtained in Experiment 2 was similar to a neutral point procedure described by Harris (1970). To determine this neutral point, observers were shown corridor arrays with only one test grating of a spatial frequency intermediate to the adapting gratings. These test stimuli were identical to Figure 2 in all respects except that there was only one grating in each array, and it was located in either the lower or the upper region of the corridor. For each of these two arrays, observers were instructed to move to a neutral distance, at which the test grating evoked one of the aftereffect colors (e.g., green) with movement closer to the screen and the complementary color (e.g., red) with movement away from the screen. If perceived spatial frequency influences the color aftereffects, and if a grating in the upper region of the array is perceived as larger than a grating in the lower region, the neutral point settings for a grating in the upper region should be significantly further from the viewing screen than the settings for the identical grating in the lower region.

\section{Method}

Observers. Twelve undergraduate students with the characteristics specified in Experiment 1 participated in Experiment 2.

Stimuli and Design. The conditions of presentation of the adapting gratings were the same as in Experiment 1, with the following exceptions. Adaptation consisted of alternately presenting a 3-cycle/deg grating and a 6-cycle/deg grating projected through a magenta (Wratten No.34a) and a green (Wratten No. 53) gelatin filter. Color and spatial frequency of adapting gratings were counterbalanced across observers, resulting in two between-subjects adaptation conditions.

The preliminary test pattern was used again to determine the presence of a spatial frequency-contingent color aftereffect. Figures 1 and 2 were also used again to test for aftereffects contingent on perceived spatial frequency. In order to determine the neutral point for the aftereffect colors, two corridor arrays with a single 4.2-cycle/deg grating in either the lower or upper region were also included as test stimuli.

Procedure. Each observer was adapted for $60 \mathrm{~min}$ by alternately presenting the two adapting gratings for $25 \mathrm{sec}$ with intervening 5 -sec dark periods. The observers rested for $10 \mathrm{~min}$ after adaptation and then were tested for a spatial frequencycontingent color aftereffect in the same manner as in Experiment 1 . In order to be included in the test phase of Experiment 2, observers had to report aftereffect colors that were roughly complementary to each of the adaptation colors in that region of the preliminary test pattern with the same spatial frequency as the adapting grating. Four observers were replaced in the appropriate adaptation conditions for failing to meet this prerequisite.

Observers were then tested for aftereffects contingent on perceived spatial frequency. Figure 1 was presented on the viewing screen and observers were instructed to (1) move forward (or backward) until both gratings appeared to contain the same aftereffect color, (2) move backward (or forward) until both gratings appeared to contain the other aftereffect color, and (3) move to an intermediate distance at which the aftereffect colors began to switch. When Step 3 had been completed, the observer was asked to indicate which face of the cube was perceived as the front, and while the cube was in that organiza- 
tion, whether there were any aftereffect colors on the two test gratings.

The observers were then shown the corridor array containing two 4.2-cycle/deg gratings, and Steps 1-3 were repeated. Observers were again questioned to determine the presence of any aftereffect colors on the two test gratings after the completion of Step 3.

The neutral point of the aftereffects was obtained by having the observers locate the crossover distance for the aftereffect colors in the corridor array containing a single 4.2-cycle/deg grating in the lower region of the array. This process was then repeated for the corridor array containing a single grating in the upper region. Order of presentation of the two test figures was counterbalanced.

Finally, each observer was asked Questions C1, C2, and C3 from Experiment 1 to determine if there were perceived size differences between the gratings contained on Figures 1 and 2 consistent with Emmert's law.

\section{Results and Discussion}

There were no systematic differences between adaptation conditions in the pattern of color and size judgments, so the results were pooled across conditions. For the Necker cube containing two 4.2-cycle/deg gratings, 11 of the 12 observers were able to complete Postadaptation Step 3. The 12th observer did not report the presence of any aftereffect color at any distance from the viewing screen. Of the 11 observers who did complete Step 3, 9 reported different aftereffect colors on the two gratings (i.e., one test grating on the cube appeared reddish and the other grating appeared greenish). The other two observers reported one aftereffect color on one of the gratings and no aftereffect color on the other grating (i.e., the other grating appeared achromatic). The reported hue differences between the identical gratings for all 11 observers were consistent with perceived spatial frequency-contingent color aftereffects based on size differences predicted by Emmert's law.

Seven of the 12 observers were able to complete Postadaptation Step 3 for the corridor array containing two 4.2-cycle/deg gratings; the remaining five observers failed to report the presence of any aftereffect colors at any distance from the viewing screen. Of the seven observers who completed Step 3, three reported that the test gratings on the corridor array contained two different aftereffect colors and four reported that one grating contained one aftereffect color and the other grating appeared achromatic. The hues of the color reports for six of these seven observers were consistent with perceived size differences predicted by Emmert's law.

When observers were shown the two corridor arrays containing a single 4.2-cycle/deg grating, there was a significant difference between the neutral point settings for the test grating in the lower region of the array (mean $=2.67 \mathrm{~m}$ ) and those for the same grating in the upper region of the array $($ mean $=2.95 \mathrm{~m})\left[\mathrm{t}_{\mathrm{dep}}(10)=\right.$ $3.89, \mathrm{p}<.01]$.

Eleven of the 12 observers gave a response to Question $\mathrm{C} 1$ regarding perceived size differences between the two gratings on the corridor array that was consistent with Emmert's law. In addition, 11 of the 12 observers also gave a response to Question C3 regarding perceived size differences between the two gratings on the Necker cube that was consistent with Emmert's law.

The overall pattern of color reports, neutral point data, and size judgments for Experiment 2 again support the hypothesis that, under the appropriate conditions, color aftereffects can be made contingent on the perceived spatial frequency of test gratings. The most striking finding of this experiment was the presence of different aftereffect colors on two achromatic test gratings of identical retinal spatial frequency. Additionally, the neutral point data obtained in the present experiment indicated that the nature of the color aftereffects was different for gratings of identical retinal spatial frequency placed in the context of a pictorial corridor array.

Most explanations of contingent color aftereffects have in common the notion that a simple featureextraction process underlies human form perception and that the aftereffects indicate the involvement and the properties of the feature-extraction mechanisms. The findings that color aftereffects can be made contingent on perceptual organization (Uhlarik, Pringle, \& Brigell, 1977) and on perceived spatial frequency suggest problems for any explanation that is strictly tied to the spatial parameters of the adapting and test stimuli.

This is not surprising, in that a large body of research on size constancy has indicated that perception can range from being correlated with the retinal properties of objects to being correlated with the perceived properties, depending on the cues provided. Most investigations of color aftereffects have been conducted under reduced cue conditions, in that a two-dimensional bar grating is typically presented on a viewing screen in a dimly lit room. If the study of color aftereffects is to reveal the processes underlying normal vision, further research should include stimuli that are representative of cues present in the normal visual environment.

\section{REFERENCES}

HARris, C. S. Effect of viewing distance on a color aftereffect specific to spatial frequency. Psychonomic Science, 1970, 21, 350. (Abstract)

Stromeyer, C. F. Edge-contingent color aftereffects: Spatial frequency specificity. Vision Research, 1972, 12, 717-733.

Uhlarik, J., Pringle, R., \& Brigell, M. Color aftereffects contingent on perceptual organization. Perception \& Psychophysics, 1977, 22, 506-510.

\section{NOTE}

1. All of the two-alternative forced-choice data were analyzed using a binomial distribution having the following characteristics: $\mathrm{n}=24 ; \mathrm{p}=\mathrm{q}=.5$.

(Received for publication March 13, 1982.) 